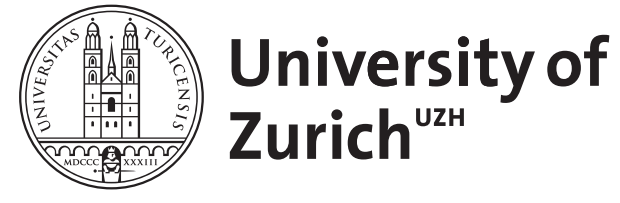

Zurich Open Repository and Archive

University of Zurich

University Library

Strickhofstrasse 39

CH-8057 Zurich

www.zora.uzh.ch

Year: 2013

\title{
Behandlungsdauer von Harnwegsinfekten bei Männern
}

\author{
Markun, Stefan
}

DOI: https://doi.org/10.1024/1661-8157/a001272

Posted at the Zurich Open Repository and Archive, University of Zurich

ZORA URL: https://doi.org/10.5167/uzh-78331

Journal Article

Accepted Version

Originally published at:

Markun, Stefan (2013). Behandlungsdauer von Harnwegsinfekten bei Männern. Praxis, 102(9):549-550.

DOI: https://doi.org/10.1024/1661-8157/a001272 


\title{
Behandlungsdauer von Harnwegsinfekten bei Männern
}

\author{
Frage: \\ Vermindert eine längere Behandlungsdauer die Rezidivrate von Harnwegsinfekten bei \\ Männern?
}

Hintergrund:

Das Auftreten von Harnwegsinfekten bei Männern wird meist von Risikofaktoren, wie Diabetes mellitus oder Prostatahyperplasie begünstigt. Entsprechend geht man bei Männern meist von einem sogenannt komplizierten Harnwegsinfekt aus. Deswegen empfehlen Fachgesellschaften bei Männern mit Harnwegsinfekten eine längere Behandlungsdauer mit Antibiotika. Die meisten Empfehlungen lauten auf 7-14 Tage. Der effektive Nutzen einer Behandlung von mehr als 7 Tagen wurde bisher jedoch noch nicht untersucht.

\section{Einschlusskriterien:}

- Männer die durch das Kriegsveteranenministerium der USA medizinisch-administrativ betreut werden

- Alle ambulanten Fälle mit Harnwegsinfekten im Jahre 2009

\section{Ausschlusskriterien:}

- Harnwegsinfekte, welche bereits 2008 aufgetreten sind und die Episode von 2009 als Rezidiv innerhalb von 30 Tagen zu werden ist

- Patienten mit Harnwegsinfekten, welche mit keinem Antibiotikum behandelt wurden

- Patienten welche mit $\geq 3$ unterschiedlichen Antibiotika behandelt wurden

\section{Studiendesign und Methode:}

Retrospektive Studie basierend auf der Analyse von administrativen Daten. Vergleich von Harnwegsinfekt-Patienten, welche $>7$ Tage antibiotisch behandelt wurden versus $\leq 7$ Tage.

\section{Studienort:}

Ganze USA

\section{Outcome:}

Pr i mär er Out come

- Effekt der Behandlungsdauer auf die frühen (<30 Tage) oder späten ( $\geq 30$ Tage) Rezidive von Harnwegsinfekten

\section{Sekundär e Out comes}

- Effekt der Behandlungsdauer auf die Infektionen mit Clostridium difficile

- Effekt anderer gemessener Variablen auf die Rezidiv Rate, sowie auf die Infektionsrate mit Clostridium difficile.

\section{Resultat:}

- Das Kriegsveteranenministerium administrierte im Jahr 2009 die ambulanten Konsultationen von 4'854'765 männlichen Patienten.

- Bei 33'336 Patienten traten ein oder mehrere Harnwegsinfekte auf, diese Patienten waren durchschnittlich 67.9 Jahre alt, die häufigsten bekannten Risikofaktoren für Harnwegsinfekte waren Diabetes mellitus (35\%), Benigne Prostatahyperplasie (33\%), sowie vormaliger Harnwegsinfekt (31\%)

- $35 \%$ der Patienten erhielten eine Behandlung von $\leq 7$ Tagen, 65\% >7 Tagen. 
- Frühe Rezidive (<30d) nach antibiotischer Behandlung ereigneten sich gesamthaft in $4.1 \%$ der Fälle, späte Rezidive ( $\geq 30$ d) in 9.9\%. In 63\% der Fälle wurde Ciprofloxacin rezeptiert, in 27\% Trimethoprim/Sulfamethoxazol, seltener Nitrofurantoin und Amoxicillin/Clavulansäure in je 6\%. 7\% der Patienten erhielten $\geq 2$ Antibiotika.

- Die unterschiedliche Behandlungsdauer hatte keinen Effekt auf die frühen Rezidive, späte Rezidive traten bei längerer Antibiotikabehandlung signifikant häufiger auf (10.8\% versus 8.4\%). Auch nach statistischer Bereinigung für die dokumentierten Risikofaktoren war eine längere Behandlungsdauer noch signifikant mit höheren Rezidiv Raten assoziiert.

- Im Vergleich mit Chinolonen traten bei Patienten, die mit Betalaktamen behandelt wurden etwa doppelt so häufig frühe Rezidive und Clostridium difficile Infektionen auf.

\section{Kommentar:}

- Dass späte Rezidive häufiger nach längerer antibiotischer Behandlung auftreten ist überraschend. Dieses Resultat wurde nach Korrektur für erfasste Risikofaktoren (Prostatahyperplasie etc.) bestätigt. Da die Analyse allerdings auf administrativen Daten beruht ist es möglich, dass diverse relevante Faktoren nicht erfasst wurden, wie z.B. das Vorhandensein eines Blasenkatheters. Patienten, welche relevante aber nicht erfasste Risikofaktoren aufweisen sind somit möglicherweise länger behandelt worden. Das vermehrte Auftreten von späten Rezidiven in der Gruppe der länger Behandelten wäre dann auch auf die nicht erfassten Risikofaktoren zurückzuführen. Die längere Behandlung wäre dann ebenso eine logische Folge von Risikofaktoren, wie die höhere Rezidivrate.

- Es wird zudem diskutiert, dass eine längere antibiotische Behandlung auch ein schwerer Eingriff in die natürliche mikrobielle Flora darstellt. Eine bakterielle Überwucherung mit pathogenen Keimen könnte auch Infektionen der Harnwege begünstigen. Am Beispiel der Clostridieninfektionen konnte dieser bekannte Effekt in dieser Studie erneut gezeigt werden.

- Ciprofloxacin war in der Studie das meist gebrauchte Antibiotikum bei Harnwegsinfekten. Es ist zu bedenken, dass die Chinolonresistenz von E. coli in der Schweiz ca. 18\% beträgt und auch bei Männern ist E. coli der am häufigsten bei Harnwegsinfekten nachgewiesene Keim. Chinolone sind in der Schweiz entsprechend nicht Antibiotika der ersten Wahl bei Harnwegsinfekten.

- Insgesamt fand sich in der Studie kein verbessertes Outcome durch eine Behandlungsdauer >7 Tage bei Männern mit Harnwegsinfekten. Zusätzlich erwiesen sich Betalaktame den üblicheren Antibiotika bei Harnwegsinfekten unterlegen aufgrund höherer Inzidenz von Rezidiven und Clostridieninfektionen.

\section{Literatur:}

Drekonja DM, Rector TS, Cutting A, Johnson JR. Urinary Tract Infection in Male Veterans: Treatment Patterns and Outcomes. Arch Intern Med. Dec 2012:1-7.

\section{Verfasser:}

Stefan Markun 